\title{
Éditorial : De la scientificité de l'anglais de spécialité
}

Editorial: On the scientific nature of ESP

\section{Anthony Saber}

\section{(2) OpenEdition}

1 Journals

Édition électronique

URL : https://journals.openedition.org/asp/5933

DOI : 10.4000/asp.5933

ISSN : 2108-6354

\section{Éditeur}

Groupe d'étude et de recherche en anglais de spécialité

\section{Édition imprimée}

Date de publication : 1 novembre 2019

Pagination : 1-8

ISSN : 1246-8185

\section{Référence électronique}

Anthony Saber, «Éditorial : De la scientificité de l'anglais de spécialité », ASp [En ligne], 76 | 2019, mis en ligne le 01 novembre 2019, consulté le 30 novembre 2021. URL : http://journals.openedition.org/ asp/5933 ; DOl : https://doi.org/10.4000/asp.5933

Ce document a été généré automatiquement le 30 novembre 2021.

Tous droits réservés 


\title{
Éditorial : De la scientificité de l'anglais de spécialité
}

\author{
Editorial: On the scientific nature of ESP
}

\author{
Anthony Saber
}

1 La publication en 2018 d'un article stimulant de Nathalie Caron et Caroline Rolland Diamond sur les fragilités du statut épistémologique de la «civilisation » en tant que secteur des études anglophones ${ }^{1}$ devrait nous conduire, puisque nous considérons l'anglais de spécialité comme une branche à part entière des études anglaises au sein du paysage universitaire français, à soulever les mêmes questionnements au sujet de notre discipline. Le lecteur nous permettra de livrer, dans cet aparté liminaire, à l'occasion de ce numéro d'automne, quelques éléments de réflexion sur ce sujet.

2 L'enjeu crucial, on le voit d'emblée, est celui de la scientificité de l'anglais de spécialité en tant que discipline. La reconnaissance de ce statut scientifique peut passer, comme l'on sait, par la publication d'un projet de recherche dans une revue à comité de lecture. En tant que vecteur de publication ayant pour ambition de contribuer à la solidité et à la crédibilité de l'anglais de spécialité, la revue ASp s'efforce de s'entourer des garanties de scientificité reposant sur des pratiques éditoriales recommandées, notamment une double évaluation anonyme par les pairs pour les soumissions reçues, assortie d'un troisième avis en cas de désaccord manifeste entre les deux premiers relecteurs. Les fiches d'évaluation détaillées issues de ce processus de relecture permettent au comité de rédaction d'obtenir des éléments d'information sur la solidité scientifique d'une soumission, notamment quant à la qualité de la problématisation proposée et à la densité des éléments de preuve présentés. Pour autant, même si elle est pratiquée par la quasi-totalité des revues scientifiques à comité de lecture aujourd'hui, le système de la double peer-review ne constitue pas à lui seul la panacée, et ne présente pas une garantie absolue de scientificité (ceux qui, parmi nous, ont pratiqué la double correction de copies dans des jurys de concours ont pu faire l'expérience directe de la grande variabilité qui peut se manifester dans l'appréciation d'un travail intellectuel). Soulignons aussi qu'en anglais de spécialité, discipline qui, en raison de sa relative jeunesse, ne s'est pas encore emparée de tous ses objets de recherche potentiels 
(l'anglais des sciences humaines et sociales ou des disciplines artistiques, par exemple, demeure très peu exploré, que ce soit sous l'angle des discours ou de l'enseignement), il peut parfois être problématique de trouver des relecteurs experts d'un sujet nouveau proposé par l'auteur d'une soumission. Il convient donc de reconnaître que, même si elle peut être considérée actuellement comme la "moins mauvaise» procédure d'appréciation des travaux scientifiques, la double relecture peut comporter des biais ; ceci a poussé certaines revues de recherche, certes en petit nombre pour l'instant, à se tourner vers des approches alternatives telles que l'open peer review ${ }^{2}$ ou de la discursive peer review ${ }^{3}$, qui méritent réflexion et mettent en perspective le processus éditorial de reconnaissance de scientificité généralement mis en œuvre aujourd'hui.

3 Au-delà des pratiques éditoriales des revues de recherche, dont on peut espérer qu'elles permettent de garantir une scientificité relative des travaux publiés, c'est avant tout la posture épistémologique d'un projet de recherche qui lui confère la fiabilité scientifique attendue. Est notamment en cause ici ce que l'on a pu appeler la posture " poppérienne » de la science contemporaine, qui, selon les recommandations du grand épistémologue Karl Popper ${ }^{4}$, doit prévoir, tout en énonçant des résultats, les conditions de leur "falsification ", c'est-à-dire formuler des énoncés qui, s'ils étaient vérifiés, invalideraient les conclusions obtenues. Cette posture n'est sans doute pas suffisamment adoptée dans les travaux de recherche en anglais de spécialité, très probablement parce qu'ils reposent, la plupart du temps, sur une démarche inductive (c'est souvent à partir d'une étude de terrain ou portant sur un corpus spécialisé, que les chercheurs en anglais de spécialité proposent des hypothèses généralisables, selon une approche que les Anglophones qualifieraient de «bottom-up»), alors que la théorie de K. Popper s'inspire beaucoup des travaux d'Einstein sur la relativité, qui procédait d'une modélisation théorique initiale destinée à être vérifiée ultérieurement par des tests de physique expérimentale permettant d'en valider ou non les principes essentiels. Dans la logique poppérienne, inspirée de la démarche d'Einstein, la science suit donc un rythme ternaire: formulation initiale d'une théorie, déduction de conséquences, mise en œuvre d'expériences vérificatrices, dont les résultats constituent potentiellement des énoncés falsificateurs pouvant invalider la théorie. Récemment encore, la découverte des ondes gravitationnelles ${ }^{5}$ venait confirmer un résultat prédit par Einstein. S'il semble patent qu'un fonctionnement de cette nature n'est pas toujours adapté aux recherches en anglais de spécialité, il n'en demeure pas moins que la posture poppérienne pourrait conférer une meilleure validité aux cadres conceptuels que nous mobilisons souvent. L'on se prend à imaginer, par exemple, un John Swales qui aurait formulé, dans son ouvrage bien connu Genre Analysis (1990), des énoncés falsificateurs au sujet de la modélisation de la structure rhétorique des introductions au sein des articles de recherche. Car si le modèle CARS (Create a Research Space) proposé par J. Swales pour décrire le feuilletage rhétorique de ces introductions en mouvements rhétoriques (moves), eux-mêmes décomposés en étapes intermédiaires (steps), est bel et bien vérifié dans de nombreuses disciplines expérimentales, sa validité apparaît bien plus relative dès que l'on aborde les disciplines des sciences humaines, comme l'économie ou la sociologie. En l'absence de ces énoncés falsificateurs, qui auraient sans doute amené J. Swales à ajouter des dimensions supplémentaires à sa théorie, de manière à ce qu'elle englobe et explique l'ensemble des phénomènes empiriquement constatables, la structure rhétorique des articles de sciences humaines et sociales en anglais demeure ainsi très largement impensée. 
4 La scientificité, ne l'oublions pas par ailleurs, n'est pas conceptualisée de la même manière dans les grands secteurs de la connaissance, que l'on décrit souvent, par commodité (car la réalité est sans doute plus complexe qu'il n'y parait), par une tripartition entre sciences exactes, sciences expérimentales et sciences humaines et sociales. Dit autrement, un énoncé scientifique n'est pas confronté aux mêmes conditions d'acceptabilité dans ces trois secteurs. Dès lors se pose la question de l'affiliation de l'anglais de spécialité à l'un ou l'autre de ces secteurs. De ce point de vue, le tableau qu'offre la discipline semble assez hétérogène : la science statistique, science exacte, est de plus en plus utilisée pour dépouiller les fréquences de termes ou de schémas phraséologiques dans les grands corpus spécialisés anglophones; les expérimentations de dispositifs pédagogiques innovants en secteur LANSAD peuvent être classées comme travaux relevant des sciences expérimentales; les approches ethnographiques des discours spécialisés anglophones, ou les études d'analyse des besoins (needs analysis) utilisent à l'évidence les outils et méthodes des sciences humaines. Il n'est donc pas aisé de définir le «type » de scientificité privilégié par l'anglais de spécialité. De fait, s'il est parfaitement légitime que les travaux d'anglais de spécialité mobilisent des cadres adaptés à l'objet de recherche dont ils s'emparent, on peut cependant s'interroger sur l'unité de la discipline, dans la mesure où elle semble disposer d'ancrages dans les trois « types » de science que nous évoquions plus haut.

5 Autre élément de réflexion sur la scientificité : celle-ci, étant ancrée dans des pratiques scientifiques de nature différente, n'est peut-être pas unique et uniforme. En effet, si l'on considère que l'activité scientifique peut se décliner en actions, qui peuvent à leur tour se résumer par des verbes de la langue naturelle (Linné se préoccupait par exemple de classer les espèces animales et végétales), il apparaît que l'anglais de spécialité, si l'on considère son périmètre d'intervention de manière assez restrictive (c'est-à-dire, grosso modo, l'analyse des discours spécialisés et des cultures qui s'y rattachent d'une part, et les travaux sur les contextes LANSAD d'autre part), n'occupe pas toute la gamme des actions scientifiques possibles. Nous avons tenté de répertorier dans le tableau 1 ci-dessous ${ }^{6}$ certaines des actions fréquemment accomplies par les scientifiques dans différents domaines. Comme on le constate, l'anglais de spécialité se préoccupe surtout, pour le moment du moins, de caractériser les phénomènes, et tend donc à se rapprocher assez exclusivement d'une scientificité descriptive. Or, on pourrait imaginer qu'il gagnerait à étendre son champ d'action scientifique, et à adopter, pour certains objets de recherche, une posture analytique. Les modèles conceptuels proposés notamment par Michel Petit (2010), Jean-Claude Bertin et al. (2010), ou Michel Van der Yeught (2016) illustrent ce type de positionnement. Quant à la scientificité de type expérimental, elle reste à notre connaissance peu répandue en anglais de spécialité, même si certains travaux de recherche-action s'en rapprochent (Mc Allister \& Belan [2014], entre autres, expérimentent l'efficacité concrète d'un dispositif hybride d'apprentissage pour l'acquisition du vocabulaire spécialisé).

Tableau 1. Typologie (exploratoire et non limitative) des « actions » scientifiques

Actions animées par une scientificité descriptive

Recueillir, inventorier (botanique, terminologie)

Classer (Linné)

Caractériser (linguistique de corpus, anglais de spécialité) 
Actions scientifiques animées par une scientificité analytique ou explicative

Expliquer, interpréter (analyse par Durkheim des déterminants sociaux du suicide, par exemple, ou encore recherche clinique en médecine)

Modéliser (modèle standard en cosmologie, théorie des cordes en physique théorique, théories économiques de Keynes, Friedmann, Piketty...)

Prédire (physique des particules de très haute énergie, théorie de la tectonique des plaques de Wegener en géoscience)

Actions scientifiques animées par une scientificité expérimentale

Mener des essais (mécanique, sciences de l'ingénieur)

Provoquer des réactions (chimie, biologie)

Vérifier des performances (sciences de l'ingénieur)

6 Cinquième élément de réflexion : la scientificité gagne à être envisagée du point de vue de la sociologie de la science, qui met en évidence son caractère relatif. Pierre Bourdieu, Paul Feyerabend ou Bruno Latour ont montré, chacun selon sa perspective, que la science est avant tout une pratique sociale traversée d'enjeux de pouvoir institutionnels. Il ne serait pas inintéressant d'appliquer leurs cadres de pensée aux travaux d'anglais de spécialité, qui ne sont pas exempts, sur certains sujets, d'une structuration en dogmes et en chapelles, attachés à leurs prérogatives et à la perpétuation de leurs paradigmes fondateurs. Si, comme l'affirme P. Feyerabend, la découverte scientifique s'opère essentiellement par un mécanisme de transgression, l'éclairage que nous procurerait une sociologie de l'anglais de spécialité (pour l'instant inexistante) permettrait sans nul doute de mieux appréhender sa scientificité.

7 Au terme de cette réflexion sommaire, qui n'a d'autre but que d'ouvrir un débat dans la communauté des anglisticiens de spécialité, il serait naturellement présomptueux de notre part de juger de la scientificité de l'anglais de spécialité de manière globale. Sans doute est-elle plus ou moins affirmée selon les travaux que l'on considère, et, on l'a vu aussi, elle dépend de nombreux paramètres, et peut être conceptualisée de différentes manières. Deux fragilités sont cependant apparentes en matière de scientificité de l'anglais de spécialité : soulignons en premier lieu la non-universalité des systèmes conceptuels de la discipline, car, de toute évidence, il existe des façons très divergentes de «faire" de l'anglais de spécialité dans l'univers de la recherche anglophone en English for Specific Purposes et en France, qui, à notre sens, a commencé à développer, à l'image de certaines sciences sociales, une "école française » de la discipline (nous pensons notamment aux travaux sur la fiction à substrat professionnel, inconnus chez les Anglo-Saxons). Or, une discipline scientifique de plein exercice a normalement vocation à l'universalité ; mais l'on voit bien que les grilles de lecture des phénomènes demeurent aujourd'hui très différentes, et ne convergent nullement: la linguistique systémique fonctionnelle, par exemple, qui inspire de très nombreux travaux anglosaxons, n'a connu qu'un succès très relatif en France. Par ailleurs, si l'on voulait raisonner de manière quelque peu paradoxale, il semble que les connaissances scientifiques en anglais de spécialité aient essentiellement progressé sur le mode de l'accrétion, par un cumul progressif de savoirs acquis au fil des travaux successifs (on peut ainsi considérer que l'article de recherche scientifique en anglais, qui a fait l'objet d'innombrables travaux, est désormais un genre discursif très bien décrit dans ses différentes dimensions - lexicales, phraséologiques, grammaticales, rhétoriques, pragmatiques, discursives...). Mais à ce jour, l'anglais de spécialité n'a jamais connu de 
véritable « révolution » scientifique, au sens où l'entend Thomas Kuhn dans son célèbre ouvrage sur La Structure des révolutions scientifiques. Comme l'on sait, T. Kuhn estime que la science progresse principalement par ruptures brutales, matérialisées par l'effondrement d'un paradigme dominant de la connaissance pour faire place à de nouvelles visions. Il n'est donc pas interdit de penser que la rupture, voire la révolution, soient consubstantielles à la scientificité, et la conditionnent même à proprement parler. " History warns us [...] that it is the customary fate of new truths to begin as heresies and to end as superstitions " affirmait le biologiste britannique Thomas Henry Huxley dans une conférence publiée en 1880 dans la revue Nature $e^{7}$ pour défendre la nouveauté de la théorie de Darwin sur l'évolution, et souligner le fait qu'elle serait rapidement acceptée de tous, avant d'être remplacée à son tour, un jour peut-être, par une théorie nouvelle. Il semble souhaitable que l'anglais de spécialité suive lui aussi ces cycles de destruction créatrice, de déclin et de renouveau, qui seraient probablement le signe de son accession au rang de discipline scientifique à part entière.

8 Si le lecteur nous a pardonné cette brève incursion dans la théorie de la science, il est désormais temps de présenter les travaux publiés dans la présente livraison d'ASp.

La réflexion sur les métaphores, qui a déjà fait l'objet de nombreuses publications en anglais de spécialité, trouve un nouveau prolongement dans le travail de Marie-Hélène Fries sur les rapports du $\mathrm{GIEC}^{8}$ (Groupe intergouvernemental d'experts sur l'évolution du climat), où elle montre la montée en puissance d'une posture éthique de plus en plus affirmée au fil du temps. Les textes du GIEC contiennent plusieurs dizaines de termes métaphoriques, souvent construits à l'aide de catachrèses, dont certains sont dotés d'une portée morale implicite. C'est notamment le cas de la métaphore du "point de bascule" (tipping point en anglais), constitutive de la théorie du réchauffement climatique: les équilibres fragiles du climat peuvent en effet être perturbés par les impulsions, mêmes très faibles, que leur infligent les comportements humains. D'emploi récent dans les rapports du GIEC, cette métaphore centrale matérialise une parole hybride, mi-scientifique, mi-militante, et résonne comme un appel à l'action sous la plume des experts climatologues.

10 Catherine Resche poursuit quant à elle sa réflexion sur les métaphores dans le domaine de la science économique, en s'intéressant aux «cycles économiques », image qui, comme l'on sait, décrit les phases successives d'expansion, de crise et de récession dont l'histoire économique est riche. Elle explique les mécanismes cognitifs à l'œuvre dans cette métaphore reprise par de nombreux économistes depuis le XIX ${ }^{e}$ siècle (c'est Willard Phillips qui formule en premier cette image en 1828 dans son manuel d'économie politique) : une veine organiciste analyse les cycles comme des "purges " de nature médicale, ou établit des parallèles avec des phénomènes naturels tels que le cycle des taches solaires analysé en 1875 par Stanley Jevons en corrélation avec les variations de l'activité agricole ; une veine mécaniste analyse les cycles sous l'angle plus abstrait de mouvements pendulaires ou vibratoires. Ces différentes images illustrent à merveille le mode fondamentalement analogique de la pensée économique, constamment à la recherche de métaphores constitutives, et le rôle heuristique des métaphores telles que celle du « cycle » dans les débats théoriques entre économistes.

11 Explorant un corpus diversifié composé d'articles de recherche, d'articles parus dans les revues professionnelles, d'annonces d'emploi et de normes professionnelles, Philippe Millot dresse une cartographie des champs sémantiques et de leurs réalisations lexicales dans une discipline non encore abordée par l'anglais de spécialité, 
à savoir la gestion de l'information et de la documentation. Si leur distribution est inégale au sein des genres de discours associés à la discipline, les termes-clés du domaine, identifiés à l'aide d'une approche hybride, mêlant détection automatique à l'aide du logiciel Wmatrix ${ }^{9}$ et étude qualitative menée à la main sur un échantillon représentatif, traduisent ce que $\mathrm{M}$. Scott et $\mathrm{C}$. Trimble désignaient comme l'aboutness d'un corpus de textes, à savoir sa focalisation thématique, que l'analyste peut décliner en catégories sémantiques (en l'espèce, l'organisationnel, le technique et le promotionnel sont les trois registres majoritaires qui ressortent de l'étude). L'originalité du travail mené par Philippe Millot tient tout autant à sa méthodologie innovante qu'à son sujet même, puisque, de l'aveu même de l'auteur, la gestion de l'information et de la documentation est un "petit » domaine spécialisé - " petit » par le nombre relativement faible d'acteurs qui y sont impliqués, par le répertoire assez restreint de textes qu'il produit, et par sa présence discrète sur la scène des spécialisations professionnelles (si on le compare à des domaines bénéficiant d'une forte visibilité comme la médecine ou le droit, par exemple). C'est certainement en s'aventurant dans ces domaines spécialisés plus confidentiels que l'anglais de spécialité trouvera de nouveaux terrains à explorer.

Le présent numéro comporte aussi deux notes pédagogiques par Alison Bouhmid et Claire Heuillard, ainsi qu'une recension par John Humbley.

La rédaction remercie chaleureusement les chercheurs qui ont accepté de relire les articles des numéros 75 et 76. Grâce à leurs commentaires, ils ont apporté une aide précieuse aux auteurs et à l'équipe éditoriale. Notre reconnaissance s'adresse à Alex Boulton, Jean-Pierre Charpy, Marion Charret-Del Bove, Trisha Dowling, Pascaline Faure, Christopher Gledhill, Charlotte Gould, John Humbley, Shaeda Isani, Sophie Moirand, Miguel Angel Campos Pardillos, Jacqueline Percebois, Elsa Pic, Robin Lee Nagano, Catherine Resche, Elisabeth Rowley-Jolivet, Isabelle Richard, JeanFrançois Sablayrolles, John Swales, Michel Van der Yeught et Christopher Williams.

\section{BIBLIOGRAPHIE}

BERTIN, Jean-Claude, Patrick GRAVÉ \& Jean-Paul NARCY-COMBES. 2010. Second Language Distance Learning and Teaching: Theoretical Perspectives and Didactic Ergonomics. Hershey : Information Science Reference.

CARON, Nathalie \& Caroline ROLLAND-DIAMOND. 2018. « Des sciences sociales en filière LLCER ou pourquoi le mot « civilisation » ne convient plus en études étrangères ». The Conversation, en ligne, 26 août 2018 <https://theconversation.com/des-sciences-sociales-en-filiere-llcer-oupourquoi-le-mot-civilisation-ne-convient-plus-en-etudes-etrangeres-94061>10.

JEVONS, William Stanley. 1875. « The solar period and the prie of corn ». In Investigations in Currency and Finance. Londres : Macmillan, 194-205.

KUHN, Thomas S. 1962. The Structure of Scientific Revolutions. Chicago : The University of Chicago Press. 
MCALLISTER, Julie \& Sophie BELAN. 2014. «L'anglais de spécialité en LEA à la croisée des domaines : étude de l'acquisition du lexique spécialisé ». ASp 66, 41-59.

PETIT, Michel. 2010. « Le discours spécialisé et le spécialisé du discours : repères pour l'analyse du discours en anglais de spécialité ». E-rea. En ligne : <http://journals.openedition.org/erea/1400>.

PHILLIPS, Willard. 1828. Manual of Political Economy. Boston : Hilliard, Gray, Little and Wilkins.

SCOTT, Mike \& Christopher TRIBBLE. 2006. Textual Patterns: Key Words and Corpus Analysis. Language Education (Vol. 22). Amsterdam \& New York : John Benjamins Publishing.

SWALES, John. 1990. Genre Analysis. English in Academic and Research Settings. Cambridge : Cambridge University Press.

VAN DER YEUGHT, Michel. 2016. « A proposal to establish epistemological foundations for the study of specialised languages ». ASp 69, 41-63.

WALKER, Richard \& Pascal ROCHA DA SILVA. 2015. « Emerging trends in peer review - a survey ». Frontiers in Neuroscience 9/168. En ligne, <https://www.ncbi.nlm.nih.gov/pmc/articles/

PMC4444765/>.

\section{NOTES}

1. Notamment en raison d'une trop grande hétérogénéité des pratiques et des objets de recherche, qui confère à la discipline, selon ces auteurs, un aspect de «bricolage ", alors qu'il conviendrait selon elles d'ancrer ses efforts de recherche dans des démarches relevant des sciences sociales.

2. Inaugurée en 1959 par la revue Current Anthropology, cette approche associe la publication d'un article accepté à la publication concomitante de plusieurs commentaires formulés par les pairs, avec possibilité de réponse publique de la part des auteurs. Pour un bref historique du système de la peer-review et de ses évolutions, voir par exemple R. Walker et P. Rocha da Silva (2015).

3. Voir la procédure de relecture tout à fait originale de la revue Behavioral and Brain Sciences <https://www.cambridge.org/core/blog/2017/09/10/behavioral-and-brain-sciences-anexample-of-discursive-peer-review/>.

4. Les conceptions de Karl Popper ont été formulées dans plusieurs ouvrages, notamment Die beiden Grundprobleme der Erkenntnistheorie (1930--1933), Logik der Forschung (1934) et Conjectures and Refutations: The Growth of Scientific Knowledge (1953).

5. Voir <https:// www.science-et-vie.com/ciel-et-espace/decouverte-des-ondesgravitationnelles-une-revolution-pour-l-astronomie-6554>

6. Cette liste n'est pas limitative, et il est clair pour nous que certaines des actions décrites peuvent se combiner ou s'enchaîner séquentiellement dans un projet de recherche donné.

7. Nature, vol. 22, numéro du 6 mai 1880. «The Coming of Age of the Origin of Species by T. H. Huxley, A lecture delivered at the Royal Institution, Friday March 19, 1880." La citation complète est " History warns us, however, that it is the customary fate of new truths to begin as heresies and to end as superstitions; and, as matters now stand, it is hardly rash to anticipate that, in another twenty years, the new generation, educated under the influences of the present day, will be in danger of accepting the main doctrines of the Origin of Species with as little reflection, and it may be with as little justification, as so many of our contemporaries, twenty years ago, rejected them. "

8. Ces Full Assessment Reports sont publiés tous les cinq ans environ depuis 1990 (1990, 1995, 2001, 2007, et 2014).

9. Voir <http://ucrel.lancs.ac.uk/wmatrix/>

10. Les sites ont été consultés le 30 septembre 2019. 


\section{AUTEUR}

\section{ANTHONY SABER}

Rédacteur en chef. anthony.saber@ens-paris-saclay.fr 